YUMS-95-08, CALT-68-1985, SWAT/67

hep-th/9503201

\title{
TOPOLOGICAL QCD
}

\author{
Seungjoon Hyun \\ Institute for Mathematical Sciences, Yonsei University \\ Seoul 120-749, Korea \\ (hyun@phya.yonsei.ac.kr) \\ and \\ Jaemo Park \\ Department of Physics, California Institute of Technology \\ Pasadena, CA 91125, USA \\ (jaemo@cco.caltech.edu) \\ and \\ Jae-Suk Park ${ }^{\dagger}$ \\ Department of Physics, University of Wales, Swansea \\ Swansea SA2 8PP, UK \\ (j.park@swansea.ac.uk)
}

We study the twisted $N=2$ supersymmetric Yang-Mills theory coupled with the hypermultiplets (TQCD). We suggest that the family of TQCD can be served as a powerful tool for studying the quantum field theoretic properties of the underlying physical theories.

March, 1995; to appear in Nucl. Phys. B

$\dagger$ Address after 1 Oct. 1995 : Institute for Theoretical Physics, University of Amsterdam, Valekenierstraat 65, 1018 XE Amsterdam. 


\section{Introduction}

The minimal $N=2$ super-Yang-Mills (SYM in short) theory can be twisted to define the topological Yang-Mills (TYM in short) theory [1]. The underlying asymptotically free physical theory has two different limits; the weakly coupled ultraviolet and the strongly coupled infrared limits. The path integrals of the twisted theory can be computed in both limits corresponding to the two different scaling limits. The ultraviolet limit of the theory gives the original cohomological description of the Donaldson invariants [2]. On the other hand, the infrared limit of the theory gives an entirely different viewpoint of the Donaldson invariants. This physical realization of the Donaldson invariants provides a powerful and genuine new viewpoint on the invariants of the smooth structure on four-manifolds [3] [4].

Recently, Seiberg and Witten studied the strongly coupled infrared limit of the minimal $N=2$ SYM theory [5]. Based on the resulting low-energy effective theory, Witten introduced new four-manifold invariants (the Seiberg-Witten invariants), which is the dual description of the Donaldson invariants, and determined the Donaldson invariants completely for Kähler surfaces with $b_{2}^{+} \geq 3$ 四. This new approach turns out to be extremely powerful in many respects $[7][8]$. The Seiberg-Witten monopole theory can be viewed as the twisted version? of the $N=2$ super-Maxwell theory with one massless hypermultiplet [4], which arises as the low energy effective theory of the minimal $N=2$ SYM theory [9]. It is very surprising that the abelian theory with matter describes the highly non-trivial four-manifold invariants after twisting.

In their second paper [9], Seiberg and Witten studied the strongly coupled infrared limit of the $N=2$ supersymmetric $S U(2)$ Yang-Mills theories coupled with hypermultiplets in the fundamental representation. An interesting new feature, besides from the many physical implications, is that there exists a critical theory with exactly vanishing $\beta$-function. The asymptotically free non-critical theories seem to belong to the same universality class as certain massive deformations of the critical theory. Furthermore, the critical theory has shown to have the full $S L(2, \mathbb{Z})$ symmetry exchanging electric and magnetic charges analogous to Montonen-Olive duality [10] [11], as refined and tested in [12], of the $N=4$ theory.

1 The variation of the Donaldson invariants on Kähler surface with $b_{2}^{+}=1$ is studied in 6 .

2 This is not rigorously true as we shall see later. 
One can naturally believe the above features are general properties of the $N=2$ supersymmetric gauge theories. This leads to the program of the classification of the theory up to the universality class and the duality.

In this paper, we study the twisted $N=2 \mathrm{SYM}$ theories coupled with the hypermultiplets on a compact oriented simply connected 3 Riemann 4-manifold $X$. The crucial observation is that the twisting procedure in general (including the minimal case) should always involve the $\operatorname{spin}^{c}$ structure on $X$. The appearance of the $\operatorname{spin}^{c}$ structure in the topological twisting of the $N=2 \mathrm{SYM}$ theory coupled with the $N=2$ matters is a special new feature which adds very rich flavors to the TQCD. The other crucial property is, as expected, that the theory without matter is independent of the choice of the spin ${ }^{c}$ structure used in the twisting. We refer to the theory as the topological QCD (TQCD) and the resulting topological invariants as the Donaldson-Seiberg-Witten (DSW) invariants or the monopole invariants. This invariants have some similarities with the spin polynomials proposed by Pidstrigach and Tyurin [14][15] who first used non-abelian $\operatorname{spin}^{c}$ Dirac operator to define smooth invariants.

For a given compact connected semi-simple gauge group, we have a family of the monopole invariants associated with arbitrary representations, whose data define the underlying asymptotically free or scale invariant theory, as well as with arbitrary spin ${ }^{c}$ structures on $X$. Of course the cohomological definition of the DSW invariants is based on the weakly coupled ultraviolet limit of the underlying $N=2$ supersymmetric QCD. More importantly, we suggest that the family of TQCD can be served as powerful new tool for studying the quantum field theoretic properties of the underlying physical theories.

This paper is organized as follows; In sect. 2, we review the $N=2$ supersymmetric YangMills theory coupled with hypermultiplets. In Sect. 3, we twist the hypermultiplets and define the topological QCD. We discuss the meaning of fermionic zero-modes and calculate the ghost number anomaly (the dimension of the moduli space). In Sect. 4, we study the topological observables and their correlation functions which define the DSW invariants. Finally we add some remarks on the the original Seiberg-Witten monopole equation and on the future prospects of the TQCD in Sect. 5. Up to Sect. 4, we restrict our attentions to the theory with one hypermultiplet. In the final section, we will briefly consider more general cases and their possible applications to the underlying physical theories.

3 The restriction for the simply connected case is just for convenience. A detailed account for Kähler surface will be discussed in our forthcoming paper 13. 
Many properties of TQCD as a cohomological theory can be trivially established by adopting the similar arguments of Witten on TYM theory. Hence, we will freely refer to the original paper of Witten [1]. This paper has been grown out from our efforts to understand the Seiberg-Witten theory [4] [5]. We are also motivated by Witten's suggestion that one can generalize the Seiberg-Witten equation to define a new family of four-manifold invariants [16.

We should note that the twisting of $N=2$ hypermultiplets with arbitrary gauge group was previously studied by Anselmi and Fré [17]. 1 . They use the $\sigma$-model interpretation of spin-0 bosons of a hypermultiplet while we use $\operatorname{spin}^{c}$ structures in twisting those bosons. However, the resulting equations obtained from the two approaches coincide with each other for a hyperkähler manifold [18]. Furthermore, they worked out the twisting of the most general $N=2$ supersymmetric theories including gravity [17]. After finishing this paper, a paper on a construction of topological action for the abelian Seiberg-Witten monopoles appeared [19].

\section{The $N=2$ Supersymmetric QCD}

\subsection{The Physical Action and Supersymmetry}

To begin with, we briefly review the $N=2$ supersymmetric Yang-Mills theory coupled with hypermultiplets. We generally follow the notation of Wess and Bagger [20] as well as Seiberg and Witten's papers [5][9]. We consider a compact connected simple gauge group $G$. We adopt the anti-hermitian convention of the Lie algebra generators.

The Lorentz symmetry $K$ is locally isomorphic to $S U(2)_{L} \times S U(2)_{R}$. In addition to $K$, the $N=2$ SYM theory may have the internal symmetry $U(2)_{I}$. The instanton breaks $U(2)_{I}$ to a subgroup whose connected component is isomorphic to $S U(2)_{I} \times U(1)_{\mathcal{R}} \cdot U(1)_{\mathcal{R}}$ is anomalous unless the theory is scaling (conformal) invariant. Its charge is denoted as a quantum number $U$. The global symmetry of the theory is

$$
H=S U(2)_{L} \times S U(2)_{R} \times S U(2)_{I}
$$

4 We are grateful to D. Anselmi for pointing this out after the first version of this paper. 
The supercharges $Q_{\alpha}{ }^{i}$ and $Q_{\dot{\alpha} i}$ transform under $H$ as $\left(\frac{1}{2}, 0, \frac{1}{2}\right)^{-1}$ and $\left(0, \frac{1}{2}, \frac{1}{2}\right)^{+1}$, where the superscript denotes the $U$ charge. The minimal action consists of the $N=2$ vector multiplets in the adjoint representations;

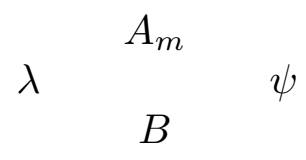

Note that $\lambda=\lambda_{\alpha}^{1}, \psi=\lambda_{\alpha}^{2}, \bar{\lambda}=\bar{\lambda}_{1}^{\dot{\alpha}}$ and $\bar{\psi}=\bar{\lambda}_{2}^{\dot{\alpha}}$. The quantum numbers for the fields under $H$ are

$$
\begin{array}{lll}
\lambda=\left(\frac{1}{2}, 0, \frac{1}{2}\right)^{+1}, & \psi=\left(\frac{1}{2}, 0, \frac{1}{2}\right)^{+1}, & B=(0,0)^{+2} \\
\bar{\lambda}=\left(0, \frac{1}{2}, \frac{1}{2}\right)^{-1}, & \bar{\psi}=\left(0, \frac{1}{2}, \frac{1}{2}\right)^{-1}, & \bar{B}=(0,0)^{-2}
\end{array}
$$

The $N=2$ supersymmetric action is given by

$$
\begin{aligned}
h^{2} L_{Y M}= & -\frac{1}{4} F_{m n}^{a} F_{a}^{m n}-i \bar{\lambda}_{\dot{\alpha} i}^{a} \bar{\sigma}^{m \dot{\alpha} \alpha} D_{m} \lambda_{\alpha a}{ }^{i}-D_{m} \bar{B}^{a} D^{m} B_{a} \\
& -\frac{i}{\sqrt{2}} \lambda^{\alpha i a}\left[\bar{B}, \lambda_{\alpha i}\right]_{a}-\frac{i}{\sqrt{2}} \bar{\lambda}_{\dot{\alpha}}{ }^{i a}\left[B, \bar{\lambda}^{\dot{\alpha}}{ }_{i}\right]_{a}-\frac{1}{2}[\bar{B}, B]^{2},
\end{aligned}
$$

where $h^{2}$ denotes the coupling constant. The supersymmetry transformation is

$$
\begin{aligned}
\delta A_{m} & =i \xi^{\alpha i} \sigma_{m \alpha \dot{\alpha}} \bar{\lambda}_{i}^{\dot{\alpha}}-i \lambda^{\alpha i} \sigma_{m \alpha \dot{\alpha}} \bar{\xi}^{\dot{\alpha}}{ }_{i} \\
\delta \lambda_{\alpha}{ }^{i} & =\sigma_{\alpha}^{m n}{ }_{\alpha} \xi_{\beta}{ }^{i} F_{m n}+\sqrt{2} i \sigma_{\alpha \dot{\alpha}}^{m} D_{m} B \bar{\xi}^{\dot{\alpha} i}+[B, \bar{B}] \xi_{\alpha}{ }^{i} \\
\delta \bar{\lambda}_{\dot{\alpha} i} & =-\bar{\xi}_{\dot{\beta} i} \bar{\sigma}^{m n \dot{\beta}} F_{m n}+\sqrt{2} i \xi^{\alpha i} \sigma_{\alpha \dot{\alpha}}^{m} D_{m} \bar{B}-[B, \bar{B}] \bar{\xi}_{\dot{\alpha} i} \\
\delta B & =\sqrt{2} \xi^{\alpha i} \lambda_{\alpha i} \\
\delta \bar{B} & =\sqrt{2} \bar{\xi}_{i}^{\dot{\alpha}} \bar{\lambda}_{\dot{\alpha}}{ }^{i}
\end{aligned}
$$

In addition to the vector multiplets one can couple the $N=2$ supersymmetric matter fields called the hypermultiplets [21]. 2 . To couple the hypermultiplet, one should specify a

5 In the coupled theory the transformation law (2.5) for the $N=2$ vector multiplet should be changed as

$$
\begin{aligned}
\delta \lambda_{\alpha}{ }^{i a} & =\sigma_{\alpha}^{m n \beta} \xi_{\beta}^{i} F_{m n}^{a}+\sqrt{2} i \sigma_{\alpha \dot{\alpha}}^{m}\left(D_{m} B\right)^{a} \bar{\xi}^{\dot{\alpha} i}+[B, \bar{B}]^{a} \xi_{\alpha}{ }^{i}+q^{i \dagger} T^{a} q^{j} \xi_{\alpha j}-q_{j}^{\dagger} T^{a} q^{i} \xi_{\alpha}{ }^{j}, \\
\delta \bar{\lambda}_{\dot{\alpha} i}^{a} & =-\bar{\xi}_{\dot{\beta} i} \bar{\sigma}^{m n \dot{\beta}} F_{m n}^{a}+\sqrt{2} i \xi^{\alpha i} \sigma_{\alpha \dot{\alpha}}^{m}\left(D_{m} \bar{B}\right)^{a}-[B, \bar{B}]^{a} \bar{\xi}_{\dot{\alpha} i}+q_{i}^{\dagger} T^{a} q^{j} \bar{\xi}_{\dot{\alpha} j}-q_{j}^{\dagger} T^{a} q_{i} \bar{\xi}_{\dot{\alpha}}{ }^{j} .
\end{aligned}
$$

We will not use this transformation law in twisting and introduce an auxiliary field. If one twists the above transformation law one directly get the twisted transformation law that can be obtained after eliminating the auxiliary field, which will be discussed in the next section. 


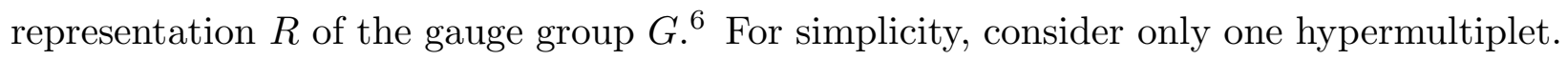
A hypermultiplet consists of two Weyl fermions $\psi_{q}$ and $\psi_{\tilde{q}}^{\dagger}$ and complex bosons $q$ and $\tilde{q}^{\dagger}$;

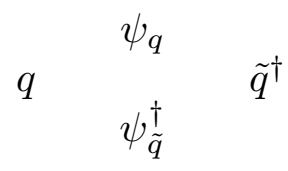

The quantum numbers of the fields under $H$ are

$$
\begin{aligned}
\psi_{q \alpha} & =\left(\frac{1}{2}, 0,0\right)^{-1}, & \bar{\psi}_{\tilde{q}}^{\dot{\alpha}} & =\left(0, \frac{1}{2}, 0\right)^{+1}, \\
\bar{\psi}_{q}^{\dot{\alpha}} & =\left(0, \frac{1}{2}, 0\right)^{+1} & \psi_{\tilde{q} \alpha} & =\left(\frac{1}{2}, 0,0\right)^{-1}, \\
q & =\left(0,0, \frac{1}{2}\right)^{0}, & q^{\dagger} & =\left(0,0, \frac{1}{2}\right)^{0}, \\
\tilde{q}^{\dagger} & =\left(0,0, \frac{1}{2}\right)^{0}, & \tilde{q} & =\left(0,0, \frac{1}{2}\right)^{0},
\end{aligned}
$$

where

$$
q^{1} \equiv q, \quad q^{2} \equiv \tilde{q}^{\dagger}, \quad q_{1}^{\dagger}=q^{\dagger}, \quad q_{2}^{\dagger}=\tilde{q}
$$

and $q_{1}=\epsilon_{12} q^{2}=-q^{2}, q_{2}=\epsilon_{21} q^{1}=q^{1}$.

The Lagrangian for the matter fields is given by

$$
\begin{aligned}
h^{2} L_{\text {Matter }}= & -\left(D_{m} q_{i}\right)^{\dagger} D^{m} q^{i}-i \bar{\psi}_{q \dot{\alpha}} \bar{\sigma}^{m \dot{\alpha} \alpha} D_{m} \psi_{q \alpha}-i \psi_{\tilde{q}}^{\alpha} \sigma_{\alpha \dot{\alpha}}^{m} D_{m} \bar{\psi}_{\tilde{q}}^{\dot{\alpha}} \\
& -\sqrt{2} \lambda^{\alpha i a} q_{i}^{\dagger} T_{a} \psi_{q \alpha}+\sqrt{2} \bar{\lambda}_{\dot{\alpha}}{ }^{i a} q_{i}^{\dagger} T_{a} \bar{\psi}_{\tilde{q}}^{\dot{\alpha}}+\sqrt{2} \bar{\psi}_{q \dot{\alpha}} T_{a} q^{i} \bar{\lambda}^{\dot{\alpha} a}{ }_{i} \\
& +\sqrt{2} \psi_{\tilde{q}}^{\alpha} T_{a} q^{i} \lambda_{\alpha i}^{a}-\sqrt{2} \psi_{\tilde{q}}^{\alpha} T_{a} \psi_{q \alpha} B^{a}+\sqrt{2} \bar{B}^{a} \bar{\psi}_{q \dot{\alpha}} T_{a} \bar{\psi}_{\tilde{q}}^{\dot{\alpha}} \\
& -q_{i}^{\dagger} T_{a} T_{b} q^{i}\left(B^{a} \bar{B}^{b}+B^{b} \bar{B}^{a}\right)+\frac{1}{2}\left(q^{i \dagger} T^{a} q^{j}+q^{j \dagger} T^{a} q^{i}\right) q_{i}^{\dagger} T_{a} q_{j} .
\end{aligned}
$$

The transformation rule for the hypermultiplet is

$$
\begin{aligned}
\delta q^{i} & =-\sqrt{2} \xi^{\alpha i} \psi_{q \alpha}+\sqrt{2} \bar{\xi}_{\dot{\alpha}}{ }^{i} \bar{\psi}_{\tilde{q}}^{\dot{\alpha}}, \\
\delta \psi_{q \alpha} & =-\sqrt{2} i \sigma_{\alpha \dot{\alpha}}^{m} D_{m} q^{i} \bar{\xi}^{\dot{\alpha}}{ }_{i}-2 T_{a} q^{i} \bar{B}^{a} \xi_{\alpha i}, \\
\delta \bar{\psi}_{\tilde{q}}^{\dot{\alpha}} & =-\sqrt{2} i \bar{\sigma}^{m \dot{\alpha} \alpha} D_{m} q^{i} \xi_{\alpha i}+2 T_{a} q^{i} B^{a} \bar{\xi}^{\dot{\alpha}}{ }_{i},
\end{aligned}
$$

and the transformation for the conjugate fields is

$$
\begin{aligned}
\delta q_{i}^{\dagger} & =-\sqrt{2} \bar{\psi}_{q \dot{\alpha}} \bar{\xi}^{\dot{\alpha}}{ }_{i}-\sqrt{2} \psi_{\tilde{q}}^{\alpha} \xi_{\alpha i}, \\
\delta \bar{\psi}_{q \dot{\alpha}} & =\sqrt{2} i \xi^{\alpha i} D_{m} q_{i}^{\dagger} \sigma_{\alpha \dot{\alpha}}^{m}-2 \bar{\xi}_{\dot{\alpha}}{ }^{i} q_{i}^{\dagger} B^{a} T_{a}, \\
\delta \psi_{\tilde{q}}^{\alpha} & =-\sqrt{2} i \bar{\xi}_{\dot{\alpha}}^{i} D_{m} q_{i}^{\dagger} \bar{\sigma}^{m \dot{\alpha} \alpha}-2 \xi^{\alpha i} \bar{B}^{a} q_{i}^{\dagger} T_{a} .
\end{aligned}
$$

6 We denotes $T_{a}$ as the generator of the gauge group in the representation $R$, and $\left[T_{a}, T_{b}\right]=$ $f^{a b c} T_{c}$. 


\section{Topological QCD}

Throughout this paper we consider a simply connected compact oriented smooth Riemann four-manifold $X$ endowed with the Riemann metric $g_{\mu \nu}$. To be precise, we recall the mathematical set-up. Let $G$ be a compact, connected simple Lie group with Lie algebra $\mathfrak{g}$. We consider a principal $G$-bundle $P$ over an oriented compact simply connected Riemann four-manifold $X$. We denote $\mathfrak{g}_{P}$ for the bundle of the Lie algebras associated to the adjoint representation. Picking an arbitrary (linear) representation $R$ of $G$, we get a vector bundle $E$ over $X$ associated with $P$ and the representation $R$. We denote $\tilde{E}$ the conjugate (or dual) vector bundle of $E$ which is the associated bundle with $P$ and the representation $\tilde{R}$ conjugate to $R$. Then, the adjoint bundle $\mathfrak{g}_{P}$ is a real sub-bundle of the endomorphism bundle $\operatorname{End}(E)=E \otimes \tilde{E}$. If $G=S U(2)$, as an example, $\mathfrak{g}_{P}$ consists of skew-adjoint trace-free endomorphisms.

\subsection{Twisting}

The twisting procedure of the minimal rigid $N=2$ SYM theory is explained very clearly in Witten's papers [1] [3]. Since the supercharges transform, under the original global symmetry group $H$, as the spinors, the global (rigid) supersymmetric theory does not exist on the non-parallelizable space. The twisting can be described as taking a diagonal subgroup $S U(2)_{R^{\prime}}$ of $S U(2)_{R} \times S U(2)_{I}$ in $H$, and regard

$$
K^{\prime}=S U(2)_{L} \times S U(2)_{R^{\prime}}
$$

as the rotation group instead of $K$. Under $K^{\prime}$, the supercharges transform as $(1 / 2,1 / 2) \oplus$ $(0,1) \oplus(0,0)$. Then we take the $(0,0)$ component of the supercharge which transforms as scalar, i.e., $Q=\varrho^{\dot{\alpha} i} Q_{\dot{\alpha} i}$, as the charge of global supersymmetry. The resulting theory, called the TYM theory, exists on any oriented Riemann manifold. The twisted transformation law is given by

$$
\begin{aligned}
& \delta A_{\mu}=i \varrho \lambda_{\mu}, \\
& \delta \lambda_{\mu}=-\varrho D_{\mu} \phi \\
& \delta \chi_{\mu \nu}=\varrho H_{\mu \nu}, \quad \delta H_{\mu \nu}=i \varrho\left[\phi, \chi_{\mu \nu}\right], \\
& \delta \phi=0, \\
& \delta \bar{\phi}=i \varrho \eta, \quad \delta \eta=\varrho[\phi, \bar{\phi}],
\end{aligned}
$$


where the fields $\left(A_{\mu}, \lambda_{\mu}, \phi, \chi_{\mu \nu}, H_{\mu \nu}, \bar{\phi}, \eta\right)$ have the $U$-numbers $(0,1,2,-1,0,-2,-1)$. Let $T_{\varepsilon}$ (field) denote the variation of a field in a gauge transformation generated by an infinitesimal parameter $\varepsilon$. One finds

$$
\left(\delta_{\varrho} \delta_{\varrho^{\prime}}-\delta_{\varrho^{\prime}} \delta_{\varrho}\right)(\text { field })=T_{\varepsilon}(\text { field })
$$

where $\varepsilon^{a}=-2 i \varrho \varrho^{\prime} \cdot \phi^{a}$.

In terms of the new global symmetry $K^{\prime}$, the hypermultiplet transforms as follows;

$$
\begin{aligned}
\psi_{q \alpha} & =\left(\frac{1}{2}, 0\right)^{-1}, & \bar{\psi}_{q}^{\dot{\alpha}} & =\left(0, \frac{1}{2}\right)^{+1} \\
\psi_{\tilde{q} \alpha} & =\left(\frac{1}{2}, 0\right)^{-1}, & \bar{\psi}_{\tilde{q}}^{\dot{\alpha}} & =\left(0, \frac{1}{2}\right)^{+1}, \\
q^{\dagger} & =\left(0, \frac{1}{2}\right)^{0}, & q & =\left(0, \frac{1}{2}\right)^{0}, \\
\tilde{q}^{\dagger} & =\left(0, \frac{1}{2}\right)^{0}, & \tilde{q} & =\left(0, \frac{1}{2}\right)^{0} .
\end{aligned}
$$

Note that all the complex bosons transform as the right-handed spinor fields under $K^{\prime}$. The appearance of the spinor fields after twisting is the new feature of the TQCD. At first sight, one might conclude that the TQCD exists only on spin manifolds.

To illuminate this point, we should look at the twisting procedure more closely. As explained in [1] [3], the use of $K^{\prime}$, instead of $K$ to generate rotations one should replace the standard stress tensor $T$ by a modified stress tensor $T^{\prime}$. This may amount to coupling the untwisted theory with external gauge fields by gauging $S U(2)_{I}$ and considering diagonal correlators with the $S U(2)_{I}$ gauge fields related to the right-handed spin connections $\omega_{R}$. However, in the non-spin manifolds, the above procedure is valid only on local region and can not be defined consistently at everywhere in the manifold. Then, it implies that the twisting in general is impossible for a non-spin manifold even for the minimal theory. However, this is not true. Instead we can use the $\operatorname{spin}^{c}$ structure which exists on arbitrary oriented Riemann 4-manifolds. In fact, the twisting procedure should involve the $\operatorname{spin}^{c}$ structure rather than the spin structure.

The tangent space $T X$ of a compact oriented Riemann 4-manifold $X$ has the structure group $S O(4)$. To define the spinor fields everywhere in $X$, one should be able to lift $S O(4)$

7 We used the argument in [22] where the twisting procedure as well as other approaches to the cohomological filed theories in general are explained in details.

8 For an extensive review on the spin geometry, the readers can consult the excellent book [23]. 
to $\operatorname{Spin}(4)=S U(2) \times S U(2)$. The obstruction of defining a spin structure is measured by the second Stifel-Whitney class $w_{2}(X) \in H^{2}(X ; \mathbb{Z} / 2)$. The spin structure does not exist unless $w_{2}(X)=0$. Instead, we consider any characteristic element $\mathfrak{c}$ such that $\mathfrak{c} \in H^{2}(X ; \mathbb{Z})$ and $\mathfrak{c} \equiv w_{2}(X) \bmod 2$ which defines a $\operatorname{spin}^{c}$ structure of $X$. A $\operatorname{spin}^{c}$ structure defines a pair of rank two hermitian vector bundle $W_{\mathfrak{c}}^{+}$and $W_{\mathfrak{c}}^{-}$such that $\operatorname{det}\left(W_{\mathfrak{c}}^{ \pm}\right)=L_{\mathfrak{c}}^{2}$ where $c_{1}\left(L_{\mathfrak{c}}^{2}\right)=\mathfrak{c} .9$ One can write

$$
W_{\mathfrak{c}}^{+}=S^{+} \otimes L_{\mathfrak{c}}
$$

where $S^{+}$and $L_{\mathfrak{c}}$ are both possibly non-existing square roots of some bundles. However, their tensor product exists everywhere. A different integral lift of $w_{2}(X) \in H^{2}(X ; \mathbb{Z} / 2)$ defines a different $\operatorname{spin}^{c}$ structure $\mathfrak{c}^{\prime}$ on $X$. Changing the $\operatorname{spin}^{c}$ structure 10 on $X$ by an $c_{1}(\zeta) \in H^{2}(X ; \mathbb{Z})$

$$
\mathfrak{c}^{\prime}-\mathfrak{c}=2 c_{1}(\zeta)
$$

amounts to twisting the given $\operatorname{spin}^{c}$ bundle by the associated line bundle $\zeta$,

$$
W_{\mathfrak{c}}^{+}=S^{+} \otimes L_{\mathfrak{c}} \rightarrow W_{\mathfrak{c}}^{+} \otimes \zeta=S^{+} \otimes L_{\mathfrak{c}} \otimes \zeta=W_{\mathfrak{c}^{\prime}}^{+}
$$

where $\operatorname{det}\left(W_{\mathfrak{c}^{\prime}}^{+}\right)=L_{\mathfrak{c}^{\prime}}^{2}=L_{\mathfrak{c}}^{2} \otimes \zeta^{2}$. Of course $\mathfrak{c}^{\prime}$ is again a characteristic. Physically this amounts to say that one can have many different choices of the topological twisting.

Now we can proceed the twisting procedure using the $\operatorname{spin}^{c}$-connection instead of the spin connection. Here the $\operatorname{spin}^{c}$-connection means the usual spin connection as well as the connection of the line bundle $L_{\mathfrak{c}}^{2}$ associated with a characteristic $\mathfrak{c}$. The soul effect of the twisting using the $\operatorname{spin}^{c}$ is that the Dirac operator is twisted by the connection on $L_{\mathfrak{c}}^{2}$. One may ask why the original TYM theory does not refer to the $\operatorname{spin}^{c}$ structure. One obvious observation is that there are no fields which transform as the spinor under $K^{\prime}$. Later, it will become clear that the twisted minimal theory (the original TYM) theory is actually independent of the choice of the $\operatorname{spin}^{c}$ structure. On the other hand, the twisting of the $N=2$ hypermultiplet depends on the choice of the $\operatorname{spin}^{c}$. This fact endows the TQCD with very rich structures. Furthermore one should view that the original TYM theory has a hidden symmetry generated by the variation of the $\operatorname{spin}^{c}$ structure. This observation has a deeper implication.

9 We will follow the physical notation of Witten[ [4].

10 Since we are considering the simply connected case, this is the only way of changing the $\operatorname{spin}^{c}$. 
To describe the topological transformation laws, one sets

$$
\xi^{\alpha i}=0
$$

and replace the internal indices $i, j, .$. of $S U(2)_{I}$ by another $S U(2)_{R}$ index $\dot{\beta}$ 迎

$$
\bar{\xi}^{\dot{\alpha} i}=\bar{\xi}^{\dot{\alpha} \dot{\beta}}=-\varepsilon^{\dot{\alpha} \dot{\beta}} \varrho
$$

where $\varrho$ is an anticommuting parameter. From (2.10) and (2.11) we have 12

$$
\begin{aligned}
\delta q^{\dot{\alpha}} & =-\varrho \bar{\psi}_{\tilde{q}}^{\dot{\alpha}}, & & \delta \bar{\psi}_{\tilde{q}}^{\dot{\alpha}}=-i \varrho \phi^{a} T_{a} q^{\dot{\alpha}}, \\
\delta q_{\dot{\alpha}}^{\dagger} & =-\varrho \bar{\psi}_{q \dot{\alpha}}, & \delta \bar{\psi}_{q \dot{\alpha}} & =i \varrho q_{\dot{\alpha}}^{\dagger} \phi^{a} T_{a},
\end{aligned}
$$

where we set

$$
B=\frac{i}{2 \sqrt{2}} \phi, \quad \begin{aligned}
& \sqrt{2} \bar{\psi}_{\tilde{q}}^{\dot{\alpha}} \rightarrow \bar{\psi}_{\tilde{q}}^{\dot{\alpha}}, \\
& \sqrt{2} \bar{\psi}_{q \dot{\alpha}} \rightarrow \bar{\psi}_{q \dot{\alpha}},
\end{aligned}
$$

One finds

$$
\left(\delta_{\varrho} \delta_{\varrho^{\prime}}-\delta_{\varrho^{\prime}} \delta_{\varrho}\right)(\text { field })=T_{\varepsilon}(\text { field })
$$

as expected.

On the other hand, the naive topological transformations

$$
\begin{gathered}
\delta \psi_{q \alpha} \approx-i \varrho \sigma_{\alpha \dot{\alpha}}^{\mu} D_{\mu} q^{\dot{\alpha}} \\
\delta \psi_{\tilde{q}}^{\alpha} \approx i \varrho D_{\mu} q_{\dot{\alpha}}^{\dagger} \sigma^{\mu \dot{\alpha} \alpha}
\end{gathered}
$$

where we use the normalization,

$$
\psi_{q \alpha} \rightarrow \sqrt{2} \bar{\psi}_{q \alpha}, \quad \psi_{\tilde{q} \alpha} \rightarrow \sqrt{2} \psi_{\tilde{q} \alpha}
$$

11 This procedure leads to the so called bispinor. In general one can regard the bispinor as a special case of the spinor carrying an external index. This point and related topics are very clearly and extensively discussed in the chapters 14 and 15 of ref. 24].

12 Note that the untwisted bosonic field $q$ is a $E$-valued scalar. After the topological twisting that replaces $q^{i}$ by $q^{\dot{\beta}}, q$ becomes a section of $\left(S^{+} \otimes L_{\mathfrak{c}}\right) \otimes E$ for a spin ${ }^{c}$ structure $\mathfrak{c}$. In the mathematics literature, taking a certain tensor product to an original bundle is usually referred as "twisting". Thus, it is legitimate to refer to the procedure as the twisting by a $\operatorname{spin}^{c}$ structure. 
do not satisfy the relation (3.12) in off-shell. This is the typical situation and can be resolved by introducing the auxiliary spinor fields $X_{q \alpha}$ and $X_{\tilde{q}}^{\alpha}$ with $U=0$;

$$
\begin{aligned}
\delta \psi_{q \alpha} & =-i \varrho \sigma_{\alpha \dot{\alpha}}^{\mu} D_{\mu} q^{\dot{\alpha}}+\varrho X_{q \alpha}, \\
\delta \psi_{\tilde{q}}^{\alpha} & =i \varrho D_{\mu} q_{\dot{\alpha}}^{\dagger} \bar{\sigma}^{\mu \dot{\alpha} \alpha}-\varrho X_{\tilde{q}}^{\alpha} .
\end{aligned}
$$

Then one can find

$$
\begin{aligned}
\delta X_{q \alpha} & =i \varrho \phi^{a} T_{a} \psi_{q \alpha}-i \varrho \sigma_{\alpha \dot{\alpha}}^{\mu} D_{\mu} \bar{\psi}_{\tilde{q}}^{\dot{\alpha}}+\varrho \sigma^{\mu}{ }_{\alpha \dot{\alpha}} \lambda_{\mu}^{a} T_{a} q^{\dot{\alpha}} \\
\delta X_{\tilde{q}}^{\alpha} & =i \varrho \psi_{\tilde{q}}^{\alpha} \phi^{a} T_{a}-i \varrho D_{\mu} \bar{\psi}_{q \dot{\alpha}} \bar{\sigma}^{\mu \dot{\alpha} \alpha}+\varrho q_{\dot{\alpha}}^{\dagger} \bar{\sigma}^{\mu \dot{\alpha} \alpha} \lambda_{\mu}^{a} T_{a} .
\end{aligned}
$$

One can check that the algebra is closed.

Before we leaves this section, we should emphasize that the Dirac operator $\not D=\sigma^{\mu} D_{\mu}$ acts on the $\operatorname{spin}^{c}$ bundle twisted by $E$,

$$
\sigma^{\mu} D_{\mu}: \Gamma\left(W_{\mathfrak{c}}^{+} \otimes E\right) \rightarrow \Gamma\left(W_{\mathfrak{c}}^{-} \otimes E\right)
$$

The square of the Dirac operator is given by the Weitzenböck formula

$$
\not D^{*} \not D=\nabla^{*} \nabla-F_{A}^{+}+\frac{i}{2} p+\frac{1}{4} R
$$

where $F_{A}^{+}$is the self-dual part of the gauge field strength and $p$ denotes Clifford multiplication by the curvature 2-form on $\operatorname{det}\left(W_{\mathfrak{c}}^{+}\right)=L_{\mathfrak{c}}^{2}$ and $R$ denotes the scalar curvature of the metric.

\subsection{Topological Action}

In general, the action functional of cohomological theory can be written as a $Q$ commutator. To motivate the correct formula we recall the familiar action functional of TYM theory. The topological action $S_{T}$ can be written as

$$
S_{T}=k / h^{2}-i\left\{Q, V_{T}\right\}
$$

where $k$ denotes the instanton number

$$
k=\frac{1}{8 \pi^{2}} \int \operatorname{Tr}\left(F_{A} \wedge F_{A}\right),
$$


and $V_{T}$ is given by

$$
V_{T}=\frac{1}{h^{2}} \int d^{4} x \sqrt{g}\left[\chi_{a}^{\mu \nu}\left(H_{\mu \nu}^{a}-i F_{\mu \nu}^{+a}\right)-\frac{1}{2} g^{\mu \nu}\left(D_{\mu} \bar{\phi}\right)_{a} \lambda_{\nu}^{a}+\frac{1}{8}[\phi, \bar{\phi}]_{a} \eta^{a}\right] .
$$

We have

$$
\begin{array}{r}
S_{T}=k / h^{2}+\frac{1}{h^{2}} \int d^{4} x \sqrt{g}\left[\left(H_{a}^{\mu \nu}-\frac{i}{2} F_{a}^{+\mu \nu}\right)\left(H_{\mu \nu}^{a}-\frac{i}{2} F_{\mu \nu}^{+a}\right)+\frac{1}{4} F_{a}^{+\mu \nu} F_{\mu \nu}^{+a}\right. \\
-i \chi_{a}^{\mu \nu}\left[\phi, \chi_{\mu \nu}\right]^{a}+\chi_{a}^{\mu \nu}\left(d_{A} \lambda\right)_{\mu \nu}^{+a}+\frac{i}{2} g^{\mu \nu}\left(D_{\mu} \eta\right)_{a} \lambda_{\nu}^{a}-\frac{1}{2} g^{\mu \nu}\left(D_{\mu} \bar{\phi}\right)_{a}\left(D_{\nu} \phi\right)^{a} \\
\left.-\frac{i}{2} g^{\mu \nu}\left[\lambda_{\mu}, \bar{\phi}\right]_{a} \lambda_{\nu}^{a}+\frac{i}{8}[\phi, \eta]_{a} \eta^{a}+\frac{1}{8}[\phi, \bar{\phi}]_{a}[\phi, \bar{\phi}]^{a}\right] .
\end{array}
$$

A crucial property of the above action is that it is supersymmetric in arbitrary orientable Riemann four-manifold. This looks obvious but actually a highly nontrivial property since the above action directly comes from the supersymmetric action defined on the flat space. In general when verifying supersymmetry in curved space, one meets the commutator of covariant derivative and the Riemann tensor appears. However, the only appearance of the commutator of covariant derivatives is on the fields which transform as the scalars [1]. Note also that there are no fields which transform as the spinor under $K^{\prime}$. Thus, neither the Riemann tensor nor the curvature tensor associated to the $\operatorname{spin}^{c}$ structure appear. Consequently, the TYM theory is well defined for arbitrary oriented Riemann four-manifold as well as completely independent of the spin ${ }^{c}$ structure used to define the twisting procedure. That is, the $\operatorname{spin}^{c}$ structure is completely decoupled from the theory.

Some properties of the $V_{T}$ are i) gauge invariance as well as invariance under $K^{\prime}$, ii) all the fields are in the adjoint representation, iii) the net $U$-number is ${ }^{\prime}-1^{\prime}$, iv) the non-degeneracy of the kinetic terms.

Actually, the above four properties almost completely determine the possible form of the $V_{M}$ such that the total action functional can be written as

$$
S=S_{T}+S_{M}=k / h^{2}-i\left\{Q, V_{T}+V_{M}\right\}=k / h^{2}-i\{Q, V\}
$$

A useful choice of $V_{M}$ is

$$
\begin{aligned}
V_{M}=\frac{1}{h^{2}} \int d^{4} x \sqrt{g}[ & -i \chi_{a}^{\mu \nu} q^{\dagger} \bar{\sigma}_{\mu \nu} T^{a} q+\left(X_{\tilde{q}}^{\alpha} \psi_{q \alpha}+\psi_{\tilde{q}}^{\alpha} X_{q \alpha}\right) \\
& \left.+i\left(q_{\dot{\alpha}}^{\dagger} \bar{\phi}_{a} T^{a} \bar{\psi}_{\tilde{q}}^{\dot{\alpha}}+\bar{\psi}_{q \dot{\alpha}} \bar{\phi}_{a} T^{a} q^{\dot{\alpha}}\right)\right] .
\end{aligned}
$$


This form can be easily seen from the Yukawa coupling of the underlying theory.

We should remind the reader that $\chi_{\mu \nu}$ is a self-dual tensor field in the adjoint representation. Thus only the quantities transforming as self-dual tensor and in the adjoint representation can couple to $\chi_{\mu \nu}$. This becomes more transparent if we combine $V_{T}$ and $V_{M}$,

$$
V=\frac{1}{h^{2}} \int d^{4} x \sqrt{g}\left[\chi_{a}^{\mu \nu}\left[H_{\mu \nu}^{a}-i\left(F_{\mu \nu}^{+a}+q^{\dagger} \bar{\sigma}_{\mu \nu} T^{a} q\right)\right]+\ldots\right.
$$

The above expression is completely sensible. Since $q \in \Gamma\left(W_{\mathfrak{c}}^{+} \otimes E\right)$ and $q^{\dagger} \in \Gamma\left(\bar{W}_{\mathfrak{c}}^{+} \otimes \tilde{E}\right)$, the product $q \otimes q^{\dagger}$ lies in

$$
W^{+} \otimes_{\mathfrak{c}} E \otimes \bar{W}_{\mathfrak{c}}^{+} \otimes \tilde{E} \sim \Omega^{0}(\operatorname{End}(E)) \oplus \Omega_{+}^{2}(\operatorname{End}(E))
$$

where $\Omega^{0}(\operatorname{End}(E))$ and $\Omega_{+}^{2}(E n d(E))$ denote the spaces of $E n d(E)$-valued zero-forms and $\operatorname{End}(E)$-valued self-dual-two-forms respectively. Of course $F^{+}$also lies in (the real subspace of $) \Omega_{+}^{2}(\operatorname{End}(E))$.

After small computations, we have

$$
\begin{aligned}
S_{M}=\frac{1}{h^{2}} & \int d^{4} x \sqrt{g}\left[-i H_{a}^{\mu \nu} q^{\dagger} \bar{\sigma}_{\mu \nu} T^{a} q-2 X_{\tilde{q}}^{\alpha} X_{q \alpha}+i X_{\tilde{q}}^{\alpha} \sigma^{\mu}{ }_{\alpha \dot{\alpha}} D_{\mu} q^{\dot{\alpha}}+i D_{\mu} q_{\dot{\alpha}}^{\dagger} \bar{\sigma}^{\mu \dot{\alpha} \alpha} X_{q \alpha}\right. \\
& -i \chi_{a}^{\mu \nu} \bar{\psi}_{q} \bar{\sigma}_{\mu \nu} T^{a} q+i \chi_{a}^{\mu \nu} q^{\dagger} \bar{\sigma}_{\mu \nu} T^{a} \bar{\psi}_{\tilde{q}}-i D_{\mu} \bar{\psi}_{q \dot{\alpha}} \bar{\sigma}^{\mu \dot{\alpha} \alpha} \psi_{q \alpha}-i \psi_{\tilde{q}}^{\alpha} \sigma^{\mu}{ }_{\alpha \dot{\alpha}} D_{\mu} \bar{\psi}_{\tilde{q}}^{\dot{\alpha}} \\
& +2 i \psi_{\tilde{q}}^{\alpha} \phi_{a} T^{a} \psi_{q \alpha}-2 i \bar{\psi}_{q \dot{\alpha}} \bar{\phi}_{a} T^{a} \bar{\psi}_{\tilde{q}}^{\dot{\alpha}}+q_{\dot{\alpha}}^{\dagger} \lambda_{\mu a} T^{a} \bar{\sigma}^{\mu \dot{\alpha} \alpha} \psi_{q \alpha}+\psi_{\tilde{q}}^{\alpha} \sigma^{\mu}{ }_{\alpha \dot{\alpha}} \lambda_{\mu a} T^{a} q^{\dot{\alpha}} . \\
& \left.+q_{\dot{\alpha}}^{\dagger} \eta_{a} T^{a} \bar{\psi}_{\tilde{q}}^{\dot{\alpha}}-\bar{\psi}_{q \dot{\alpha}} \eta_{a} T^{a} q^{\dot{\alpha}}-q_{\dot{\alpha}}^{\dagger} T^{a} T^{b}\left(\phi_{a} \bar{\phi}_{b}+\phi_{b} \bar{\phi}_{a}\right) q^{\dot{\alpha}}\right] .
\end{aligned}
$$

The total action functional $S=S_{T}+S_{M}$ is given by the sum of Eq. (3.22) and Eq. (3.26). If one carefully compares the untwisted action, (Eq. (2.4)+Eq. (2.9)), with the twisted action $S$, one can see that both do in fact look identical at least for the flat Euclidean 4-manifolds. In addition to the gauge symmetry, the classical action functional has the manifest $U$-number symmetry (the ghost number symmetry). The situation concerning this $U$-number symmetry is much like the one in the original TYM theory. 
One can eliminate the auxiliary fields $H_{\mu \nu}, X_{q \alpha}$ and $X_{\tilde{q}}^{\alpha}$ from the action functional. The relevant terms in the action $S$ is

$$
\begin{array}{r}
S_{0}=\frac{1}{h^{2}} \int d^{4} x \sqrt{g}\left[\left(H_{a}^{\mu \nu}-\frac{i}{2}\left(F_{a}^{+\mu \nu}+q^{\dagger} \bar{\sigma}^{\mu \nu} T_{a} q\right)\right)\left(H_{\mu \nu}^{a}-\frac{i}{2}\left(F_{\mu \nu}^{+a}+q^{\dagger} \bar{\sigma}_{\mu \nu} T^{a} q\right)\right)\right. \\
+\frac{1}{4}\left(F_{a}^{+\mu \nu}+q^{\dagger} \bar{\sigma}^{\mu \nu} T_{a} q\right)\left(F_{\mu \nu}^{+a}+q^{\dagger} \bar{\sigma}_{\mu \nu} T^{a} q\right)-2 X_{\tilde{q}}^{\alpha} X_{q \alpha} \\
\left.+i X_{\tilde{q}}^{\alpha} \sigma^{\mu}{ }_{\alpha \dot{\alpha}} D_{\mu} q^{\dot{\alpha}}+i D_{\mu} q_{\dot{\alpha}}^{\dagger} \bar{\sigma}^{\mu \dot{\alpha} \alpha} X_{q \alpha}\right] .
\end{array}
$$

One can eliminate $H_{\mu \nu}$ by setting

$$
H_{a}^{\mu \nu}=\frac{i}{2}\left(F_{a}^{+\mu \nu}+q^{\dagger} \bar{\sigma}^{\mu \nu} T_{a} q\right)
$$

We will use the notation that

$$
s=F_{a}^{+\mu \nu}+q^{\dagger} \bar{\sigma}^{\mu \nu} T_{a} q
$$

The Gaussian integration over $X_{\tilde{q}}^{\alpha}$ and $X_{q \alpha}$ is equivalent to the squaring, $\frac{1}{2}|k|^{2}$, where

$$
k=\sigma^{\mu} D_{\mu} q=\not D q
$$

Using the Weitzenböck formula (3.18), the action $S_{0}$ in (3.27) becomes 13

$$
\begin{aligned}
S_{0}=\frac{1}{h^{2}} \int d^{4} x \sqrt{g}[ & \left.\frac{1}{4}|s|^{2}+\frac{1}{2}|k|^{2}\right] \\
=\frac{1}{h^{2}} \int d^{4} x \sqrt{g}[ & +\frac{1}{4} F_{a}^{+\mu \nu} F_{\mu \nu}^{+a}+\frac{1}{4}\left(q^{\dagger} \bar{\sigma}^{\mu \nu} T_{a} q\right)\left(q^{\dagger} \bar{\sigma}_{\mu \nu} T^{a} q\right)+\frac{1}{2} g^{\mu \nu} D_{\mu} q_{\dot{\alpha}}^{\dagger} D_{\nu} q^{\dot{\alpha}} \\
& \left.+\frac{1}{8} R\left(q_{\dot{\alpha}}^{\dagger} q^{\dot{\alpha}}\right)+\frac{i}{4} p_{\dot{\alpha} \dot{\beta}}\left(q^{\dot{\alpha} \dagger} q^{\dot{\beta}}+q^{\dot{\beta} \dagger} q^{\dot{\alpha}}\right)\right] .
\end{aligned}
$$

The resulting action is $Q$ invariant after changing the transformation law for $\chi_{\mu \nu}$ to

$$
\delta \chi_{\mu \nu}^{a}=\frac{i}{2} \varrho\left(F_{\mu \nu}^{+a}+q^{\dagger} \bar{\sigma}_{\mu \nu} T^{a} q\right)
$$

13 The absence of the mixed term, $\sim F^{\mu \nu} q^{\dagger} \bar{\sigma}_{\mu \nu} q$, actually originated from the underlying untwisted theory, (Eq. (2.4) +Eq. (2.9)). The new feature of the twisted theory is the appearance of the terms proportional to $p$ and $R$. These new data precisely indicate the property of the topological twisting of the hypermultiplets. 
Similarly the eliminations of $X_{\tilde{q}}^{\alpha}$ and $X_{q \alpha}$ give

$$
\begin{aligned}
\delta \psi_{q \alpha} & =-\frac{i}{2} \varrho \sigma_{\alpha \dot{\alpha}}^{\mu} D_{\mu} q^{\dot{\alpha}}, \\
\delta \psi_{\tilde{q}}^{\alpha} & =\frac{i}{2} \varrho D_{\mu} q_{\dot{\alpha}}^{\dagger} \bar{\sigma}^{\mu \dot{\alpha} \alpha} .
\end{aligned}
$$

Thus the fixed point locus, $\delta \chi_{\mu \nu}=\delta \psi_{q \alpha}=\delta \psi_{\tilde{q}}^{\alpha}=0$, of the theory is the space of solutions of the following equations

$$
\begin{aligned}
F_{\mu \nu}^{+a}+q^{\dagger} \bar{\sigma}_{\mu \nu} T^{a} q & =0, \\
\sigma^{\mu} D_{\mu} q & =0,
\end{aligned}
$$

which is the non-Abelian version of the Seiberg-Witten monopole equations. The solutions of the equation (3.34) are of course the minimum energy solutions of $S_{0}$.

Due to the fixed point theorem of Witten, the path integral reduces to the fixed point locus. Equivalently, in the semi-classical limit $h^{2} \rightarrow 0$ (which is exact) the path integral receives the dominant contribution from the minimum action configurations of $S_{0}$ which corresponds to the solution space of the equation (3.34). We refer the space of solution modulo gauge symmetry as the SW moduli space. The moduli space of TQCD can be specified by

$$
\mathcal{M}(X, G, R, k, \mathfrak{c})
$$

where $X$ is the underlying oriented simply connected Riemann 4-manifold, $G$ is the gauge group, $R$ denotes the representation of $G$ carried by the hypermultiplets, $k$ is the instanton number and $\mathfrak{c}$ is the $\operatorname{spin}^{c}$ structure on $X$ used to define the topological twisting. If we consider the theory with no hypermultiplets, the moduli space is the moduli space of ASD connections which will be denoted by $\mathcal{M}(X, G, k)$.

\subsection{The Classical Moduli Space}

Here the notion of classical or quantum moduli space is used in the sense of the SeibergWitten paper [9]. It is useful to collect the bosonic terms of the action,

$$
\begin{aligned}
S_{\text {bose }}=k / h^{2}+\frac{1}{h^{2}} \int d^{4} x & \sqrt{g}\left[\frac{1}{4} F_{a}^{+\mu \nu} F_{\mu \nu}^{+a}+\frac{1}{4}\left(q^{\dagger} \bar{\sigma}^{\mu \nu} T_{a} q\right)\left(q^{\dagger} \bar{\sigma}_{\mu \nu} T^{a} q\right)+\frac{1}{2} g^{\mu \nu} D_{\mu} q_{\dot{\alpha}}^{\dagger} D_{\nu} q^{\dot{\alpha}}\right. \\
+ & \left.\frac{1}{8} R\left(q_{\dot{\alpha}}^{\dagger} q^{\dot{\alpha}}\right)+\frac{i}{4} p_{\dot{\alpha} \dot{\beta}}\left(q^{\dot{\alpha} \dagger} q^{\dot{\beta}}+q^{\dot{\beta} \dagger} q^{\dot{\alpha}}\right)-\frac{1}{2} g^{\mu \nu}\left(D_{\mu} \bar{\phi}\right)_{a}\left(D_{\nu} \phi\right)^{a}\right] .
\end{aligned}
$$


The theory has another important fixed point equations

$$
\delta \lambda_{\mu}=-\varrho D_{m} \phi=0, \quad \delta \eta=\varrho[\phi, \bar{\phi}]=0 .
$$

The existence of a solution $\phi$ of the above equation means that the gauge connection is reducible. Then the bundle reduces to the direct sum of certain line bundles. Physically, it corresponds to the flat direction of the superpotential and the gauge group $G$ spontaneously breaks down to its maximal torus. As far as concerning the gauge symmetry, the theory with one hypermultiplet has only two phases, the unbroken phase of gauge symmetry and the broken phase to its maximal torus.

Furthermore the action functional as well as the equation (3.34) are invariant under the global vector $U(1)$ symmetry (circled action) generated by the scaling of spinor with $e^{i \theta}$. Unlike the gauge symmetry which is a redundant symmetry that should be fixed, this $U(1)$ symmetry is the true symmetry of the theory. This suggests that the minimum action configuration space is degenerated. Furthermore this $U(1)$ symmetry has the fixed points. One fixed point is obviously the configuration $q=0$. The solution space of (3.34) or the minimum action configuration space is the moduli space of $G$-ASD connections modulo gauge symmetry. The other fixed points are in the Coulomb phase that a nonzero solution of $\phi$ of (3.37) exist. If we consider the case that $G=S U(2)$ and $R$ is the fundamental representation, the gauge symmetry spontaneously breaks down to $U(1)$ and the vector bundle $E$ reduces to a sum of line bundles $E=\zeta_{1} \oplus \zeta_{2}$. Then, for example, $q=\left(q_{1}, q_{2}=0\right)$ is a fixed point since the $U(1)$ action on $q_{1}$ can be undone by the $U(1)$ gauge transformation. Then the monopole equation (3.34) becomes the abelian monopole equation. We will return this point later which turns out to be crucial. If we consider the theory with more hypermultiplets, this global symmetry will be enhanced accordingly.

In this paper, we will not try to analyze the detailed structure of the solution space. We just would like to remark on the relation with the classical moduli space of the underlying untwisted theory [5]. To establish an analogy, we consider a $K 3$ surface and the metric with vanishing scalar curvature. Since $K 3$ surfaces are spin manifolds, we simplify the argument by choosing trivial $\operatorname{spin}^{c}$ structure for twisting, i.e. $\mathfrak{c}=0$. Then the only possibility of the solution for (3.34) is just $q=0, D_{\mu} \phi=0$ and $F^{+}=0$. Thus there are two possibilities for the pairs $(q, A)$; i) $q=0$ and $A$ is an ASD connection, ii) $q=0$ and $A$ is an abelian instanton (Coulomb Phase). If we increase the number of hypermultiplets there can be other types of solutions which allow the certain non-vanishing combination of spinors as 
a solution. This immediately requires the spinor to be a covariant constant. (Of course this is allowed only for the hyperKähler manifold). This branch is referred as the Higgs branch. What we tried to say is that the vanishing theorem arguments are analogous to studying the classical moduli space of the underlying physical theory. But the relations between two seems to be not that close because of the appearance of $R$ and $p$. Instead, we will demonstrate later that the study of the minimum energy solution space of TQCD is more closely related to the quantum moduli space of the underlying theory studied in the second paper of Seiberg and Witten [9].

\subsection{The Fermionic Zero-Modes}

Now we discuss the geometric meaning of the fermionic zero-modes. Here we refer to the fermionic zero-modes by the fields with odd $U$-number (the ghost number) following the conventions of the usual TYM theory. The $\chi_{\mu \nu}$ equation of motion, modulo the gauge symmetry, gives

$$
\left(d_{A} \lambda\right)_{\mu \nu}^{+a}-i \bar{\psi}_{q} \bar{\sigma}_{\mu \nu} T^{a} q+i q^{\dagger} \bar{\sigma}_{\mu \nu} T^{a} \bar{\psi}_{\tilde{q}}=0
$$

The $\psi_{\tilde{q}}^{\alpha}$ equation of motion, modulo the gauge symmetry, gives

$$
i \sigma_{\alpha \dot{\alpha}}^{\mu} D_{\mu} \bar{\psi}_{\tilde{q}}^{\dot{\alpha}}-\sigma_{\alpha \dot{\alpha}}^{\mu} \lambda_{\mu}^{a} T_{a} q^{\dot{\alpha}}=0
$$

These two equations (3.38) and (3.39) are precisely the linearization of the equations (3.34) by identifying the infinitesimal variations $\left(\delta A, \delta q, \delta q^{\dagger}\right)$ with $\left(\lambda, i \bar{\psi}_{\tilde{q}}, i \bar{\psi}_{q}\right)$. Note that all $\lambda, \bar{\psi}_{\tilde{q}}$ and $\bar{\psi}_{q}$ have the $U$-number (the ghost number) 1 . Furthermore, the $\eta$ equation of motion gives

$$
D_{\mu} \lambda_{a}^{\mu}+i q_{\dot{\alpha}}^{\dagger} T_{a} \bar{\psi}_{\tilde{q}}^{\dot{\alpha}}+i \bar{\psi}_{q \dot{\alpha}} T_{a} q^{\dot{\alpha}}=0
$$

which expresses the fact that $\left(\lambda, i \bar{\psi}_{\tilde{q}}, i \bar{\psi}_{q}\right)=\left(\delta A, \delta q, \delta q^{\dagger}\right)$ is orthogonal to the gauge directions. Thus the zero-modes of $\left(\lambda, \bar{\psi}_{\tilde{q}}, \bar{\psi}_{q}\right)$ are the tangent vectors of $\mathcal{M}$. In the generic situation, the number of the zero-modes $\left(\lambda, \bar{\psi}_{\tilde{q}}, \bar{\psi}_{q}\right)$ corresponds to the real dimension of the moduli space $\mathcal{M}$. Of course, the moduli space is the moduli space of ASD connections in the absence of the hypermultiplets.

The dimension of the moduli space can be easily calculated by applying the AtiyahSinger index theorem. After twisting the complex boson $q(\tilde{q})$ can be viewed as a section 
of $W^{+} \otimes E\left(W^{+} \otimes \tilde{E}\right)$. By defining $\delta_{A}=d_{A}^{+} \oplus d_{A}^{*}$, the above equations (3.38), (3.39) and (3.40) can be summarized as

$$
\delta_{A} \oplus \not D: \Omega^{1}\left(\mathfrak{g}_{P}\right) \oplus\left(W_{\mathfrak{c}}^{+} \otimes E\right) \rightarrow \Omega^{0}\left(\mathfrak{g}_{P}\right) \oplus \Omega_{+}^{2}\left(\mathfrak{g}_{P}\right) \oplus\left(W_{\mathfrak{c}}^{-} \otimes E\right)
$$

Thus the real virtual dimension is given by the sum of the index of $\delta_{A}$ and the twice of the usual Dirac index of $\not D$. The index of $\delta_{A}$ is well known, and gives the virtual dimension of the moduli space $\mathcal{M}(X, G, k)$ of anti-self-dual connections,

$$
\begin{aligned}
\operatorname{index}\left(\delta_{A}\right) & =\operatorname{dim}(\mathcal{M}(X, G, k))=p_{1}\left(\mathfrak{g}_{P}^{\mathbb{C}}\right)-\frac{\operatorname{dim}(G)}{2}(\chi+\sigma) \\
& =4 c_{2}(\operatorname{adj}) k(P)-\frac{\operatorname{dim}(G)}{2}(\chi+\sigma)
\end{aligned}
$$

where $\chi$ and $\sigma$ denote the Euler characteristic and the signature of $X$, and $k(P)$ denotes the instanton number

$$
k(P)=\frac{1}{8 \pi^{2}} \int_{X} \operatorname{Tr} F_{A} \wedge F_{A} .
$$

The index of the twisted Dirac operator associated to a $\operatorname{spin}^{c}$ structure $\mathfrak{c}$ is given by

$$
\operatorname{index}(\not D)=\int_{X} e^{\frac{1}{2} c_{1}\left(L_{\mathrm{c}}^{2}\right)} \operatorname{ch}(E) \wedge \hat{A}(X)
$$

where the trace inside $\operatorname{ch}(E)$ should be taken in the representation $R$ which the hypermultiplet carries. The rank of $E$ is identical to the dimension of the representation $r k(E)=\operatorname{dim}(R)$. We have 14

$$
\begin{aligned}
\operatorname{index}(\not D) & =\frac{1}{2} \int_{X}\left(c_{1}^{2}(E)-2 c_{2}(E)\right)+\frac{r k(E)}{2} c_{1}^{2}\left(L_{\mathfrak{c}}\right)+\int_{X} c_{1}\left(L_{\mathfrak{c}}\right) \wedge c_{1}(E)-\frac{r k(E)}{24} \int_{X} p_{1}(X) \\
& =-2 T(R) k(P)+\frac{r k(E)}{2} c_{1}^{2}\left(L_{\mathfrak{c}}\right)+\int_{X} c_{1}\left(L_{\mathfrak{c}}\right) \wedge c_{1}(E)-\frac{r k(E)}{8} \sigma .
\end{aligned}
$$

Putting everything together we have the real virtual dimension of the moduli space

$$
\begin{aligned}
\operatorname{dim} \mathcal{M}(X, G, R, k, \mathfrak{c})= & \operatorname{dim} \mathcal{M}(X, G, k)+2 \operatorname{index}(\not D) \\
= & 4\left(c_{2}(a d j)-T(R)\right) k(P)+\operatorname{dim}(R) c_{1}^{2}\left(L_{\mathfrak{c}}\right)+2 \int_{X} c_{1}\left(L_{\mathfrak{c}}\right) \wedge c_{1}(E) \\
& -\frac{\operatorname{dim}(G)}{2}(\chi+\sigma)-\frac{\operatorname{dim}(R)}{4} \sigma .
\end{aligned}
$$

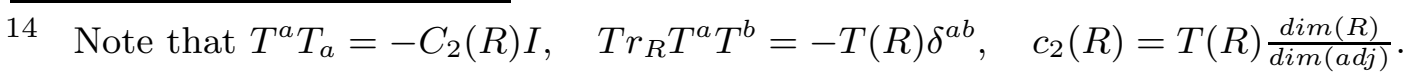




\section{The Observables and the Correlation Functions}

\subsection{The Observables}

The partition function or the correlation functions of topological observables in TYM theory are the differential topological invariants (the Donaldson invariants) of the smooth four-manifold. It is straightforward to show that the corresponding quantities in TQCD are also differential topological invariants. For simplicity we consider the simply connected four-manifolds. We also restrict our attention to the the $S U(2)$ case with hypermultiplet in the fundamental representation. The generalization for the other groups and representations should be straightforward.

The topological observables of the original TYM theory is given by

$$
\begin{aligned}
\Theta(x) & =\frac{1}{8 \pi^{2}} \operatorname{Tr} \phi(x)^{2} \\
O_{\Sigma} & =\frac{1}{4 \pi^{2}} \int_{\Sigma} \operatorname{Tr}\left(i \phi F+\frac{1}{2} \lambda \wedge \lambda\right),
\end{aligned}
$$

where $\Sigma \in H_{2}(X ; \mathbb{Z})$. The geometrical meaning of the observables is well-known. The observables $\Theta$ and $\Omega_{\Sigma}$ also serve as topological observables in the TQCD.

Since there are no additional fields, coming from the hypermultiplet, which is $Q$ invariant, one may think that there is no new additional topological observables. However, one can find that the combination,

$$
i \phi^{a} q_{\dot{\alpha}}^{\dagger} T_{a} q^{\dot{\alpha}}+\bar{\psi}_{q \dot{\alpha}} \bar{\psi}_{\tilde{q}}^{\dot{\alpha}}
$$

is $Q$-invariant. Note that the above combination carries the ghost number 2 similar to the observables $O_{\Sigma}$. Note that the two-form $\operatorname{Tr}\left(i \phi F+\frac{1}{2} \lambda \wedge \lambda\right)$ is $Q$-invariant up to an exact term which leads to the fact that $O_{\Sigma}$ is $Q$-invariant and depends only on the homology class of $\Sigma \in H_{2}(X ; \mathbb{Z})$. However the above combination is absolutely $Q$-invariant and transforms as a scalar similar to the observables $\Theta$. One can change the combination to transform as a vector, a second rank tensor, etc. In any case, the above observable does not depend on $H_{2}(X ; \mathbb{Z})$ at all and trivial in the $Q$-cohomology. In fact, the term (4.2) is a part of the bare mass term of the hypermultiplet. 


\subsection{The Correlation Functions}

The partition function of the theory is formally defined as

$$
Z=\frac{1}{\operatorname{vol}(\mathcal{G})} \int \mathcal{D} Y \exp (-S)
$$

where $\mathcal{G}$ is the group of the gauge transformations and $\mathcal{D} Y$ denotes the path integral measure. We will consider only the generic case that there are only fermionic zero-modes of $\left(\lambda, i \bar{\psi}_{\tilde{q}}, i \bar{\psi}_{q}\right)$ which correspond to the tangent vectors of the moduli space. Though the action $S$ has the global $U$-number symmetry at the classical level, the path integral measure is not invariant under $U$ due to the zero-modes of $\left(\lambda, i \bar{\psi}_{\tilde{q}}, i \bar{\psi}_{q}\right)$ which carry $U=1$. The net violation of $U$, due to the zero-modes, is identical to the dimension $\operatorname{dim} \mathcal{M}\left(X, G, R_{i}, k, \mathfrak{c}\right)$ of the moduli space. The partition function can be non-zero if the dimension of the moduli space is zero. By the standard analysis of any cohomological theory, the partition function reduces to an algebraic sum of the points of the moduli space up to sign. 1 .

For the generic choice of the metric, the moduli space is a smooth manifold with actual dimension equals to the index. Let the dimension of the moduli space be even $\operatorname{dim} \mathcal{M}\left(X, G, R_{i}, k, \mathfrak{c}\right)=2 d$. Then the correlation functions

$$
\begin{aligned}
\left\langle\Theta^{r} \prod_{i=1}^{s} O_{\Sigma_{i}}\right\rangle & =\frac{1}{\operatorname{vol}(\mathcal{G})} \int \mathcal{D} Y e^{-S} \cdot \Theta^{r} \prod_{i=1}^{s} O_{\Sigma_{i}} \\
& =e^{-k / h^{2}} \times \frac{1}{\operatorname{vol}(\mathcal{G})} \int \mathcal{D} Y e^{i\{Q, V\}} \Theta^{r} \prod_{i=1}^{s} O_{\Sigma_{i}}
\end{aligned}
$$

can be non-vanishing if and only if

$$
d=2 r+s
$$

due to the ghost number anomaly. It is straightforward to show that the above correlation functions and the partition function are metric independent at least for manifolds with $b_{2}^{+}>1$. It is also straightforward to show that the quantity

$$
\frac{1}{\operatorname{vol}(\mathcal{G})} \int \mathcal{D} Y e^{i\{Q, V\}} \Theta^{r} \prod_{i=1}^{s} O_{\Sigma_{i}}=\int_{\mathcal{M}} \widehat{\Theta} \wedge \ldots \wedge \widehat{\Theta} \wedge \widehat{O}_{\Sigma_{1} \ldots \wedge} \wedge \widehat{O}_{\Sigma_{1}},
$$

reduces to the integration of the cup-products of $\widehat{\Theta} \in H^{4}(\mathcal{M} ; \mathbb{Z})$ and $\widehat{O}_{\Sigma_{i}} \in H^{2}(\mathcal{M} ; \mathbb{Z})$ where $\widehat{\Theta}$ and $\widehat{O}_{\Sigma_{i}}$ are just the restrictions and the reductions of $\Theta$ and $O_{\Sigma_{i}}$. We will denote the above invariants as $\operatorname{DSW}_{X, G, R, k, \mathfrak{c}}\left(\Sigma_{1}, \ldots, \Sigma_{s},(p t)^{d(k)-2 s}\right)$.

15 Combining the arguments of [25] and [4], it is straightforward to define the orientation of the moduli space. 


\section{Some Prospectives}

\subsection{On the Seiberg-Witten invariants}

We consider twisted $N=2$ super-Maxwell theory with one hypermultiplet. To do the topological twisting we should choose a $\operatorname{spin}^{c}$ structure $\mathfrak{c}$. Then the monopole is a section of $W_{\mathfrak{c}}^{+} \otimes E$ where $E$ is a line bundle whose curvature is the gauge field strength of the $U(1)$ gauge symmetry. According to the famous Dirac quantization condition, the first Chern class $c_{1}(E)$ corresponds to the charge of the magnetic monopole. The formula derived by Seiberg-Witten precisely quantize the monopole charge to be an integral value [5]. That is, $c_{1}(E) \in H^{2}(X ; \mathbb{Z})$. As we reviewed before, taking the tensor products $E$ to $W_{\mathfrak{c}}^{+}$amounts to changing the $\operatorname{spin}^{c}$ structure by $c_{1}(E)$. Thus, both $W_{\mathfrak{c}}^{+}$and $W_{\mathfrak{c}}^{+} \otimes E$ are $\operatorname{spin}^{c}$ bundles associated to the different choices of the $\operatorname{spin}^{c}$ structure on $X$. In other words, they correspond to the different choice of the topological twisting. Then, it is unnecessary to specify the $U(1)$-bundle $E$ associated with the gauge group, as Witten did. Once all these are understood, our formalism precisely recovers the original Seiberg-Witten monopole equation [4] and the corresponding topological field theory for each choice of the $\operatorname{spin}^{c}$ structure or the topological twisting.16

\subsection{On a $N=4$ theory}

Now we consider the case when the hypermultiplet is in the adjoint representation. The underlying physical theory corresponds to the $N=4$ SYM theory. Interestingly enough, the dimension of the moduli space is independent of the instanton numbers,

$$
\begin{aligned}
\operatorname{dim}(\mathcal{M})_{\beta=0}= & \operatorname{dim}(\operatorname{adj}) c_{1}^{2}\left(L_{\mathfrak{c}}\right)+2 \int_{X} c_{1}\left(L_{\mathfrak{c}}\right) \wedge c_{1}(E) \\
& -\frac{\operatorname{dim}(G)}{2}(\chi+\sigma)-\frac{\operatorname{dim}(a d j)}{4} \sigma
\end{aligned}
$$

Assume that the dimension is a positive even integer $2 d$. Since the theory has vanishing $\beta$-function, we have natural a theta angle $\theta$. We turn on the theta term in the action,

$$
S \rightarrow S+\frac{i \theta}{2 \pi} k
$$

16 Precisely speaking, one obtains a perturbed version of the monopole equation, however, one can removes the perturbation. 
and introduce

$$
\tau=\frac{\theta}{\pi}+\frac{8 \pi i}{h^{2}}, \quad q=e^{2 \pi i \tau}
$$

Since the dimension of the moduli space is independent of the instanton number, one can naturally sum up the correlation function

$$
\begin{aligned}
\sum_{k}\left\langle\prod_{i=1}^{d} O_{\Sigma_{i}}^{\prime}\right\rangle_{k} & =\sum_{k}\left(\frac{1}{\operatorname{vol}(\mathcal{G})} \int \mathcal{D} Y e^{2 \pi \tau k+i\{Q, V\}} \prod_{i=1}^{d} O_{\Sigma_{i}}^{\prime}\right) \\
& =\sum_{k} q^{k} \operatorname{DSW}\left(\Sigma_{1}, \ldots \Sigma_{d}\right)_{k}
\end{aligned}
$$

According to the Montonen -Olive duality conjecture [10], the refinements of VafaWitten[12] and Seiberg-Witten[9], one can expect that the above expression is modular covariant. If we consider a manifold with $b^{+}=1$, following the suggestion of Vafa and Witten [12], one can also expect that there will be holomorphic anomaly.

At least for the one case we can confirm the expectation. Consider the $K 3$ surfaces. A $K 3$ surface is simply connected oriented spin manifold with trivial canonical line bundle. Since it has a spin structure, we can choose $c_{1}\left(L_{\mathfrak{c}}^{2}\right)=\mathfrak{c}=0$. Now we choose $G=S U(2)$, $N_{f}=1$ and $R=a d j$. . Then we have $\operatorname{dim}(\mathcal{M})=0$. Thus we have a well-defined partition function $Z_{k}$ and

$$
\sum_{k} q^{k} Z_{k}
$$

The underlying theory is in fact the $N=4$ super-Yang-Mills theory. There are three different ways of twisting and two of them were studied by Yamaron 17 26] and one of the two was studied by Vafa and Witten 12]. They also showed that the resulting theory defines a modular covariant form. Since the canonical line bundle of a $K 3$ surface is trivial, twisting does nothing. Thus, the above expression (5.5) should be also the modular covariant form.

\subsection{On the cobordism and the electro-magnetic duality}

Throughout this paper, we examined formal properties of the TQCD. In this section, we demonstrate that the TQCD can be a powerful tool to classify the $\mathrm{N}=2$ supersymmetric QCD up to the electro-magnetic duality. For the $G=S U(2)$ and $N_{f}=0,1,2,3,4$ numbers

17 One can easily check that the remaining one possibility corresponds to our case. Note also that the other two different ways of twisting are independent of the $\operatorname{spin}^{c}$ structure. 
of hypermultiplets all in the fundamental representations, this problem has been completely solved by Seiberg and Witten [5] [9]. Using the similar analysis, the $G=S U(N)$ and $N_{f}=0$ case was studied in [27] [28].

One of the possible strategy of finding the dual theory is to use the twisted versions of the various possible theories and to compare the differential-topological answers. This method was successfully used to test the $S$-duality of the $N=4 \mathrm{SYM}$ theory [12]. For example, if one studies the twisted $N=2$ super-Maxwell theory with one hypermultiplet, one would be able to see the great similarity between the basic class and the Seiberg-Witten class. However, the precise relation of the Donaldson invariants and the Seiberg-Witten invariants can not be obtained by just looking at the twisted $N=2$ super-Maxwell theory with one hypermultiplet. Furthermore, the above strategy is rather difficult and unilluminating.

Actually, one can do much better. It turns out that the TQCD with one hypermultiplet understands the strong and the weak coupling limits of the underlying physical $N=2$ supersymmetric Yang-Mills theory coupled with no matter. To be concrete, we consider the $N=2$ SYM theory with gauge group $S U(2)$. The underlying mathematical argument of the following procedure is due to Pidstrigach [29], and Tyurin [30] and Mrowka [31].

One can twist the theory to get the standard TYM theory. One consider the exact semi-classical limit of the theory and the path integral has the dominant contribution from the moduli space of $S U(2)$ ASD connections. Our goal is to find the dual theory of the underlying physical theory. One can assume that the dual theory also has a $N=2$ supersymmetric theory. One can twist the dual theory and this twisted theory is essentially characterized by the semi-classical data of the untwisted theory. The duality means that those data talk about the strong-coupling limit of the original $S U(2), N=2 \mathrm{SYM}$ theory. Of course this gives the dual description of the $S U(2)$ TYM theory.

Now we consider the $N=2 \mathrm{SYM}$ theory coupled with one hypermultiplet $\left(N_{f}=1\right)$ carrying the fundamental representation of $S U(2)$. Picking a $\operatorname{spin}^{c}$ structure $\mathfrak{c}$, one can twist the theory as we discussed. We consider the exact semi-classical limit of the twisted theory that the path integral is localized to the fixed points locus (3.32) (3.33) which is the solution space of the equations (3.34). The formal dimension of the moduli space, the space of solution modulo gauge symmetry is then

$$
\operatorname{dim} \mathcal{M}(k, R, \mathfrak{c})=8 k-\frac{3}{2}(\chi+\sigma)+\frac{1}{2} \mathfrak{c}^{2}-2 k-\frac{1}{2} \sigma
$$


On the other hand the dimension of the ASD connections is given by

$$
\operatorname{dim} \mathcal{M}(k)=8 k-\frac{3}{2}(\chi+\sigma) .
$$

Thus the codimension of $\mathcal{M}(k)$ in $\mathcal{M}(k, R, \mathfrak{c})$ is

$$
\operatorname{dim} \mathcal{M}(k, R, \mathfrak{c})-\operatorname{dim} \mathcal{M}(k)=\frac{1}{2} \mathfrak{c}^{2}-2 k-\frac{1}{2} \sigma .
$$

As we vary the instanton number $k$ the codimension also vary. However, one can choose the different topological twisting for the hypermultiplet such that,

$$
\frac{1}{2} \mathfrak{c}^{2}-2 k-\frac{1}{2} \sigma=2,
$$

the moduli space $\mathcal{M}(k, R, \mathfrak{c})$ is always two dimension higher than the moduli space $\mathcal{M}(k)$ of ASD connections.

Recall the the TQCD with one hypermultiplet has a global $S^{1}$ action on $q$. So one can form the quotient space

$$
\mathcal{M}(X, G, R, k, \mathfrak{c}) / U(1),
$$

which is one dimension higher than $\mathcal{M}(k)$. The $S^{1}$ action has an obvious fixed point $q=0$ which is the moduli space $\mathcal{M}(k)$ of ASD connections. The other fixed points is in the Coulomb phase in which the connection $A$ is reducible, $E=\zeta_{1} \oplus \zeta_{2}$, and $A$ preserves the splitting. Now spinor $q$ has the two components $q=\left(q_{1}, q_{2}\right)$. If $q_{2}=0$ then $(A, q)$ is a fixed point. $A$ induces a connection $B$ in $\zeta$ and the pair $\left(B, q_{1}\right)$ solves the (perturbed) $U(1)$ monopole equation. More precisely, one can obtain the Seiberg-Witten monopole equation for $W_{\mathfrak{c}}^{+} \otimes \zeta$, i.e., $\operatorname{det}\left(W_{\mathfrak{c}}^{+} \otimes \zeta\right)=L_{\mathfrak{c}}^{2} \otimes \zeta^{2}$.

Then, the quotient $\mathcal{M} / S^{1}$ looks like a cobordism between $\mathcal{M}_{A S D}$ and the cone on projective spaces corresponding to the reducible solutions. Thus the evaluation of the Donaldson map on the moduli space $\mathcal{M}_{A S D}$ is the same as the evaluation on the projective space. Thus the $U(1)$ Seiberg-Witten monopole theory can be an alternative description of the Donaldson theory.

The above observation has profound physical implications. Note that the topological interpretation of the path integral of the TQCD is essentially based on the weakly coupled ultraviolet limit of the underlying untwisted $N=2$ supersymmetric QCD. However, the electro-magnetic duality of the $N=2$ super-Yang-Mills theory without matter is a genuine 
quantum theoretical effect. The above example shows that the dual theory of the $N=2$ super-Yang-Mills theory may be determined by studying purely semi-classical properties of TQCD. Of course, the above duality is for the twisted theory. However, since the underlying $N=2$ super-Yang-Mills theory is asymptotically free its dual theory is a effective theory of strongly coupled infrared limit. In the large scaling limit, the Riemann manifold looks much like a flat Euclidean manifold. Thus the underlying theory for the dual description of the Donaldson theory can be viewed as the dual theory of the $N=2$ super-Yang-Mills theory. This is the reverse of the way Witten arrived in his monopole invariants from the dual theory of the physical $N=2$ super-Yang-Mills theory.

The TQCD provides much more general picture than the cobordism argument. If we consider general groups and representations it is generically impossible to fix the codimension to 2. Then, the quotient space like (5.10) does not lead to the cobordism mentioned above. However, it is always possible to express the Donaldson invariants in terms of the Seiberg-Witten invariants. In a forthcoming paper [32], we will present a explicit and general relation between the Donaldson invariants and the Seiberg-Witten invariants based on the twisted $N=2$, super-Yang-Mills theory coupled with hypermultiplet having the bare mass.

\subsection{The summary and generalization}

For a compact connected simple Lie group $G$ with the Lie algebra $\mathfrak{g}$, we consider an arbitrary sequence of representations $\left(R_{1}, R_{2}, \ldots, R_{N_{f_{c}}}\right)$ of $G$ such that

$$
a\left(R_{1}, R_{2}, \ldots, R_{N_{f_{c}}}\right)=\left(c_{2}(a d j)-\sum_{i=1}^{N_{f_{c}}} T\left(R_{i}\right)\right)=0
$$

Throughout this section, we will use the fixed sequence of representations given above.

Now we consider the sequence of the $N=2$ super-Yang-Mills theories on the flat Euclidean 4-manifold $\mathbb{R}^{4}$ coupled with $N_{f}$ number of hypermultiplets carrying the representations $R_{1}, R_{2}, \ldots, R_{N_{f}}$ where $N_{f} \leq N_{f_{c}}$. The $N=2$ super-Yang-Mills theory, in terms of the $N=1$ superspace language, consists of a vector multiplet $W_{\alpha}$ and a chiral multiplet $\Phi$ in the adjoint representation. The theories with $N_{f} \neq 0$ have additional $N_{f}$ pairs of 
conjugate chiral multiplets $Q_{i}$ and $\tilde{Q}_{i}$ in the representations $R_{i}$ and $\tilde{R}_{i}$, respectively. The one-loop $\beta$-function of the theory is given by

$$
\beta(h)=-\frac{h^{3}}{(4 \pi)^{2}} a\left(R_{1}, R_{2}, \ldots, R_{N_{f}}\right) .
$$

Thus we have the sequence of the asymptotically free theories and the critical theory $N_{f}=N_{f_{c}}$ with vanishing $\beta$-functions 18

$$
S Y M \equiv S Q C D(0) \leftrightarrow S Q C D(1) \leftrightarrow S Q C D(2) \leftrightarrow \ldots S Q C D\left(N_{f_{c}-1}\right) \leftrightarrow S Q C D\left(N_{f_{c}}\right)
$$

Associated to this, we have a sequence of inequalities

$$
a(0)>a\left(R_{1}\right)>a\left(R_{1}, R_{2}\right)>\ldots>a\left(R_{1}, R_{2}, \ldots, R_{N_{f_{c}}-1}\right)>a\left(R_{1}, R_{2}, \ldots, R_{N_{f_{c}}}\right)=0 .
$$

Now we consider the compact oriented simply connected Riemann manifold $X$. A spin ${ }^{c}$ structure $\mathfrak{c} \in H^{2}(X ; \mathbb{Z})$ is an integral lift of the Stifel-Whitney class $w_{2}(X) \in H^{2}(X ; \mathbb{Z} / 2)$, i.e. $\quad \mathfrak{c} \equiv w_{2}(X) \bmod 2$. The space $H^{2}(X ; \mathbb{R})$ of harmonic two-forms on $X$ is an $b_{2^{-}}$ dimensional flat space with signature $\left(b_{2}^{+}-b_{2}^{-}\right)$. The space $H^{2}(X ; \mathbb{Z})$ is the integral lattice in $H^{2}(X ; \mathbb{R})$. Then, the set $H_{s}^{2}(X ; \mathbb{Z})$ of all spin ${ }^{c}$ structure is an affine sublattice of $H^{2}(X ; \mathbb{Z})$. Obviously, there are $b_{2}$ independent generators $\mathfrak{s}$ of the transitive action on $S$. We consider a vector bundle $P$ on $X$ with the reduction of the structure group to $G$. We denote $\mathfrak{g}_{P}$ for the bundle of the Lie algebras associated to the adjoint representation. For the sequence $\left\{R_{i}\right\}$ of the representations we have a sequence of vector bundles $\left\{E_{i}\right\}$ over $X$ which are associated with $V$ and the sequence $\left\{R_{i}\right\}$. We denote $\left\{\tilde{E}_{i}\right\}$ the sequence of the conjugate (or dual) vector bundles of $E_{i}$. The adjoint bundle $\mathfrak{g}_{P}$ is a real sub-bundle of the endomorphism bundles $\operatorname{End}\left(E_{i}\right)=E_{i} \otimes \tilde{E}_{i}$.

To define the topological twist of the $N=2$ supersymmetric QCD with $N_{f}$ hypermultiplets, one should specify $N_{f}$ independent $\operatorname{spin}^{c}$ structures $\mathfrak{c}_{1}, \mathfrak{c}_{2}, \ldots, \mathfrak{c}_{N_{f}}$. Then we twist the sequence of the hypermultiplets with corresponding sequence of the spin ${ }^{c}$ structures. This amounts to twisting the vector bundle $E_{i}$ by the $\operatorname{spin}^{c}$ bundle $W_{\mathfrak{c}_{i}}^{+}$in the sense

18 The $\beta$-function (5.12) is exact in the all loop perturbations. There are non-perturbative instanton corrections which was studied in [33]. However, it was argued that the $\beta$-function of a critical theory vanishes exactly [9]. 
that the vector bundle $E_{i}$-valued complex boson $q_{i}$ in the $i$-th hypermultiplet should be regarded as a section of $W_{\mathfrak{c}_{i}}^{+} \otimes E_{i}$,

$$
q_{i} \in \Gamma\left(W_{\mathfrak{c}_{i}}^{+} \otimes E_{i}\right)
$$

We call the resulting topological QCD denoted by $T\left(\mathfrak{c}_{1}, \mathfrak{c}_{2}, \ldots, \mathfrak{c}_{N_{f}}\right)$ as the TQCD associated to the sequence $\mathfrak{c}_{1}, \mathfrak{c}_{2}, \ldots, \mathfrak{c}_{N_{f}}$ of the $\operatorname{spin}^{c}$ structures. We call a TQCD, $T\left(\mathfrak{c}_{1}, \mathfrak{c}_{2}, \ldots, \mathfrak{c}_{N_{f}}\right)$, critical if $N_{f}=N_{f_{c}}$ and we call a TQCD, $T_{0}$, minimal if $N_{f}=0$.

We can vary each $\operatorname{spin}^{c}$ structures independently to define another TQCD. In general we have different TQCD for the different choice of the spin $^{c}$ structures. The minimal theory $T_{0}$ is independent of the choice of the $\operatorname{spin}^{c}$ structure. The TQCD with $N_{f}$ hypermultiplets has $N_{f}$ independent choices of the $\operatorname{spin}^{c}$ structure and in each $\operatorname{spin}^{c}$ structure has $b_{2}$ independent ways of variations. Thus the space of the TQCD with $N_{f}$ hypermultiplets is $b_{2} \cdot N_{f}$ dimensional, i.e., $H_{s}^{2}(X ; \mathbb{Z}) \otimes \ldots \otimes H_{s}^{2}(X ; \mathbb{Z})$.

After twisting, we get a topological action which has a global supersymmetry. According to the fixed point theorem of Witten, the path integral reduces to an integration over the fixed point locus. The fixed point equations of the theory are

$$
\begin{aligned}
F_{\mu \nu}^{+}+q_{i}^{\dagger} \bar{\sigma}_{\mu \nu} q^{i} & =0, \\
\sigma^{\mu} D_{\mu} q^{i} & =0,
\end{aligned}
$$

where $q_{i} \in \Gamma\left(W_{\mathfrak{c}_{\mathfrak{i}}}^{+} \otimes E_{i}\right), F_{\mu \nu}^{+} \in \Omega_{+}^{2}\left(\mathfrak{g}_{P}\right)$ is the self-dual part of the curvature tensor on $P$ and

$$
\sigma^{\mu} D_{\mu}: \Gamma\left(W_{\mathfrak{c}_{\mathfrak{i}}}^{+} \otimes E_{i}\right) \longrightarrow \Gamma\left(W_{\mathfrak{c}_{\mathfrak{i}}}^{-} \otimes E_{i}\right)
$$

is the twisted Dirac operator of the $\operatorname{spin}^{c}$ structure. We denote the space of all solutions $\left(A, q^{i}\right)$ modulo gauge symmetry by

$$
\mathcal{M}\left(k, \mathfrak{c}_{1}, \ldots, \mathfrak{c}_{N_{f}}\right)
$$

where $k$ denote the instanton number. Note that $\mathcal{M}(k)$ is the moduli space ASD connections. The path integral of a theory $T\left(\mathfrak{c}_{1}, \ldots, \mathfrak{c}_{N_{f}}\right)$ then localized to $\mathcal{M}\left(k, \mathfrak{c}_{1}, \ldots, \mathfrak{c}_{N_{f}}\right)$ modulo gauge symmetry. The theory $T\left(\mathfrak{c}_{1}, \ldots, \mathfrak{c}_{N_{f}}\right)$ is a twisted version of the underlying asymptotically free $S Q C D\left(N_{f}\right)$. Thus, the cohomological description essentially rely on the ultra-violet weak coupling limit of $S Q C D\left(N_{f}\right)$. We can interpret the moduli space $\mathcal{M}\left(k, \mathfrak{c}_{1}, \ldots, \mathfrak{c}_{N_{f}}, \mathfrak{c}_{N_{f}}\right)$ characterizes the weak coupling limit of $\operatorname{SQCD}\left(N_{f}\right)$. More precisely, 
we should consider every moduli spaces $\mathcal{M}\left(k, \mathfrak{c}_{1}, \ldots, \mathfrak{c}_{N_{f}}\right)$ by varying the spin $^{c}$ structures to know about the weak coupling limit of $S Q C D\left(N_{f}\right)$. The virtual dimension of the moduli space can be easily calculated. For $c_{1}(P)=0$, we have 19

$$
\begin{aligned}
\operatorname{dim} \mathcal{M} & \left(k, \mathfrak{c}_{1}, \ldots, \mathfrak{c}_{N_{f}}\right) \\
& =4 a\left(R_{1}, R_{2}, \ldots, R_{N_{f}}\right) k-\frac{\operatorname{dim}(G)}{2}(\chi+\sigma)+\frac{1}{4} \sum_{i=1}^{N_{f}} \operatorname{dim}\left(R_{i}\right)\left(\mathfrak{c}_{\mathfrak{i}}{ }^{2}-\sigma\right)
\end{aligned}
$$

The theory has other important fixed point equations,

$$
D_{\mu} \phi=0, \quad[\phi, \bar{\phi}]=0 .
$$

If there are non-zero solution of $\phi$, the connection is reducible. Then the group $G$ spontaneously break down to its maximal torus.

The theory has additional global symmetry acting on hypermultiplets. In general the global internal symmetry always contains

$$
S^{1} \times S^{1} \times \ldots \times S^{1}
$$

where each circled action $S^{1}$ acts on $\Gamma\left(W_{\mathfrak{c}_{\mathfrak{i}}}^{+} \otimes E_{i}\right)$ by the unit modulus, i.e,

$$
q^{i} \rightarrow e^{i \theta_{i}} q^{i}, \quad i=1, \ldots, N_{f}
$$

However, if the sequence of the representations $\left(R_{1}, \ldots, R_{N_{f}}\right)$ contains the same representation the above global symmetry should be enhanced accordingly. This problem can be avoided by adding the bare mass terms for each hypermultiplets to maintain the above symmetry only. Since this is also the symmetry of the theory, one can mod out this circled action to reduce the path integral to the quotient space

$$
\mathcal{M}\left(k, \mathfrak{c}_{1}, \ldots, \mathfrak{c}_{N_{f}}\right) / T^{N_{f}}
$$

The above quotient space has very rich structures. For example, we consider a particular global phase symmetry

$$
q^{N_{f}} \rightarrow e^{i \theta} q^{N_{f}}
$$

19 Of course we should only consider the $\operatorname{spin}^{c}$ structures such that the dimension of the moduli space is non-negative. 
We have a natural fixed point of the action for $q^{N_{f}}=0$. The fixed point is the moduli space $\mathcal{M}\left(k, \mathfrak{c}_{1}, \ldots, \mathfrak{c}_{N_{f}-1}\right)$ which characterizes the theory $T\left(\mathfrak{c}_{1}, \ldots, \mathfrak{c}_{N_{f}-1}\right)$. The other fixed point of the action with $q^{N_{f}} \neq 0$ is in the (abelian) Coulomb phase. If there is a non-zero solution $\phi$, the gauge symmetry breaks down to its maximal torus. Then, it is always possible to find fixed point of the $S^{1}$ action by a suitable choice of $q^{N_{f}}$ in the representation $R_{N_{f}}$. By considering the various $S^{1}$ actions together, one can find many different fixed points which include $\mathcal{M}(k), \mathcal{M}\left(k, \mathfrak{c}_{1}\right), \ldots, \mathcal{M}\left(k, \mathfrak{c}_{1}, \ldots, \mathfrak{c}_{N_{f}-1}\right)$. In the abelian Coulomb phase, we also have a variety of the fixed points. It turns out that one can design a version of TQCD such that the path integral is localized to those various fixed points. It is also possible to express the topological quantities defined by the evaluation of the Donaldson map on the moduli space $\mathcal{M}\left(k, \mathfrak{c}_{1}, \ldots, \mathfrak{c}_{i}\right)$ as the sum of contributions of the fixed points in the Coulomb phase [32]. Thus, we can obtain a dual description of the various theories $T\left(\mathfrak{c}_{1}, \ldots, \mathfrak{c}_{N_{i}}\right)$ by examining the bigger theory.

The above picture suggests that we should reexamine the implication of the electromagnetic duality of the $N=2$ supersymmetric QCD. Naively speaking, an asymptotically free $N=2$ supersymmetric QCD, $S Q C D\left(N_{f}\right)$, and its dual theory can be embedded in another asymptotically free theory, $S Q C D\left(N_{f}+1\right)$, coupled with additional matter multiplet. More precisely, the weakly coupled ultraviolet limit and the strongly coupled infrared limit of $S Q C D\left(N_{f}\right)$ are realized as certain two-different fixed points of the weakly coupled ultraviolet limit of $S Q C D\left(N_{f}+1\right)$. Clearly, $S Q C D\left(N_{f}+1\right)$ has milder scaling dependency than $S Q C D\left(N_{f}\right)$ as the quantum field theory. To know the strongly coupled infrared limit of $S Q C D\left(N_{f}+1\right)$, one should examine the weakly coupled ultraviolet limit of $S Q C D\left(N_{f}+2\right)$ which again has milder scaling dependency. Finally, we can end up with the critical theory $S Q C D\left(N_{f_{c}}\right)$ which does not have any dependency on scaling. This critical theory, then, should be a self-dual theory. Thus, the ultimate solutions of the problems amount to solving all the critical theories. The existence of the electro-magnetic dual description of the asymptotically free $N=2$ supersymmetric QCD is originated from the self-duality or the quantum scaling invariance of the critical theory.

The self-duality of the critical theory was suggested by Witten [16] and also by Seiberg in the context of the $N=1$ theory [34]. It will be also interesting to see if our picture on the $N=2$ supersymmetric gauge theories has an application in the $N=1$ theories [34]( for a survey and further references see 35]). The details will be discussed elsewhere. 


\section{Acknowledgements}

We would like to thank T. Mrowka for an useful communication. SH and JSP would like to thank the organizers of the Jerusalem Winter School for hospitality. The work of JP is supported by U.S. Department of Energy Grant No. DE-FG03-92-ER40701. JP would like to thank the Isaac Newton Institute for hospitality. 


\section{References}

[1] E. Witten, Comm. Math. Phys. 117 (1988) 353.

[2] S. Donaldson, Topology 29 (1990) 257.

[3] E. Witten, J. Math. Phys. 35 (1994) 5101.

[4] E. Witten, Math. Research Lett. 1 (1994) 769.

[5] N. Seiberg and E. Witten, Nucl. Phys. B 426 (1994) 19.

[6] S. Hyun and J.-S. Park, Holomorphic Yang-Mills theory and the variation of the Donaldson invariants, hep-th/9503036.

[7] P. Kronheimer and T. Mrowka, The genus of embedded surfaces in the projective plane, preprint, 1994.

[8] C. Taubes, Symplectic manifolds and the Seiberg-Witten invariants, preprint, 1994.

[9] N. Seiberg and E. Witten, Nucl. Phys. B 431 (1994) 484.

[10] C. Montonen and D. Olive, Phys. Lett. B 72 (1977) 117.

[11] E. Witten and D. Olive, Phys. Lett. B 78 (1978) 97.

[12] C. Vafa and E. Witten, Nucl. Phys. B 431 (1994) 3.

[13] S. Hyun, J. Park and J.-S. Park, Topological QCD on a Kähler manifold, to appear.

[14] V. Pidstrigach and A. Tyurin, Izv. AN SSSR Ser. Math. 56:2 (1992) 279 (English translation: AMS 40 (1993) 163).

[15] A. Tyurin, The simple method of distinguishing the underlying differential structures of algebraic surfaces, alg-geom/9210003; Spin canonical invariants of 4-manifolds and algebraic surfaces, alg-geom/9406002.

[16] E. Witten, Lectures given at the Isaac Newton Institute, Dec. 1994.

[17] D. Anselmi and P. Fré, Nucl. Phys. B 404 (1993) 288; Nucl. Phys. B 416 (1994) 255.

[18] D. Anselmi and P. Fré, Gauged hyperinstantons and monopole equations, hepth/9411205.

[19] J.M.F. Labastida and M. Mariño, A topological Lagrangian for monopoles on fourmanifolds, hep-th/9503105.

[20] J. Wess and J. Bagger, Supersymmetry and Supergravity, second edition (Princeton University Press, 1992). 
[21] P. Fayet, Nucl. Phys. B 113 (1976) 135

[22] S. Cordes, G. Moore and S. Ramgoolam, Lectures on 2D Yang-Mills theory, equivariant cohomology and topological field theories, Part II, hep-th/9411210.

[23] H.B. Lawson, JR and M-L. Michelsohn, Spin geometry, (Princeton University Press, 1989).

[24] M.B. Green, J.H. Schwartz and E. Witten, Superstring theory, Vol. 2, (Cambridge University Press, 1987).

[25] S. Donaldson, J. Differ. Geom. 26 (1987) 397.

[26] J. Yamaron, Phys. Lett. B 213 (1988) 325.

[27] P. Argyres and A. Faraggi, The Vacuum structure and spectrum of $N=2$ supersymmetric $S U(N)$ gauge theory, hep-th/9411057.

[28] A. Klemm, W. Lerche, S. Yankielowicz and S. Theisen, Simple singularities and $N=2$ supersymmetric Yang-Mills theory, hep-th/9411048; On the monodromies of $N=2$ supersymmetric Yang-Mills theory, hep-th/9412158.

[29] V. Pidstrigach, Lecture given at Isaac Newton Inst. (Dec. 1994).

[30] A. Tyurin, Lecture given at Isaac Newton Inst. (Dec. 1994).

[31] T. Mrowka, private communication.

[32] S. Hyun, J. Park and J.-S. Park, N=2 supersymmetric QCD and four manifolds (I), (II)., to appear.

[33] N. Seiberg, Phys. Lett. B 206 (1988) 75.

[34] N. Seiberg, Electro-magnetic duality in supersymmetric non-abelian gauge theories, hep-th/9411149.

[35] N. Seiberg, The power of holomorphy - exact results in 4d SUSY field theories, hepth/9408013 and references therein. 